\title{
Use of Inhaled PGE1 to Improve Diastolic Dysfunction, LVEDP, Pulmonary Hypertension and Hypoxia in ARDS-A Randomised Clinical Trial*
}

\author{
Shahla Siddiqui ${ }^{1 \#}$, Nawal Salahuddin², Salwa Zubair ${ }^{3}$, Muniza Yousuf ${ }^{4}$, \\ Iqbal Azam ${ }^{5}$, Anwarul Hassan Gilani ${ }^{6}$ \\ ${ }^{1}$ Department of Anaesthesiology, Critical Care, Aga Khan University, Karachi, Pakistan; ${ }^{2}$ Department of Medicine, Pulmonary Criti- \\ cal Care, Aga Khan University, Karachi, Pakistan; ${ }^{3}$ Pharmacy Services, Aga Khan University, Karachi, Pakistan; ${ }^{4}$ Section of Cardi- \\ ology, Aga Khan University, Karachi, Pakistan; ${ }^{5}$ Statistician Community Health sciences, Aga Khan University, Karachi, Pakistan; \\ ${ }^{6}$ Department of Biological and Biomedical Sciences, Aga Khan University, Karachi, Pakistan. \\ Email: "shahlasi@yahoo.com
}

Received February $2^{\text {nd }}, 2013$; revised March $10^{\text {th }}, 2013$; accepted March $18^{\text {th }}, 2013$

\begin{abstract}
Introduction: We wished to see the effects of inhaled PGE1 on diastolic dysfunction, left ventricular end diastolic pressure (LVEDP), pulmonary hypertension and hypoxia in ARDS patients. Methods: This is a randomized, prospective, clinical trial conducted in the main adult intensive care unit of a tertiary care University hospital. A total of 67 patients were recruited. Inclusion criteria included all adult patients with a P/F ratio $<200$ and/or Pulmonary Artery systolic (Pa) pressures of $>35 \mathrm{mmHg}$ on Pulmonary artery catheter or suspected on clinical grounds. A transthoracic echo was performed to record the diastolic function, LVEDP and Pa pressures. Subsequently patients were randomized by a block computerized randomization to either cases $(n=34)$ or controls $(n=33)$. Cases received nebulised PGE1 over 30 minutes in the ICU and normal saline was administered to controls blindly. Following this the echo and arterial blood gases were repeated. Our primary outcomes were an improvement in diastolic function and P/F ratio of greater than $20 \%$ and a decrease in pulmonary pressure and LVEDP of $>20 \%$. Results: At baseline, mean diastolic dysfunction was grade II, with a mean LVEDP of $>15$ and the $\mathrm{PaO}_{2} / \mathrm{FiO}_{2}$ ratio was $148.38 \pm 60.05$ with a mean pulmonary artery pressure of $81.35 \pm 16.91$. Inhaled PGE1 was followed by an improvement in diastolic dysfunction (grade I, $\mathrm{p}=0.001$ ) with a resulting improvement in LVEDP $(12+/-2, \mathrm{p}=0.001)$ as well as Pa pressures $(97.09 \pm 30.06, \mathrm{p}=0.04)$ and a non significant improvement in $\mathrm{PaO}_{2} / \mathrm{FiO}_{2}$ ratio $(161.45 \pm 77.52$, $\mathrm{p}=0.21)$. There were no side effects observed in any patients. Conclusion: Our study shows that there is a significant improvement in diastolic dysfunction, LVEDP and Pa pressures after administration of nebulised PGE1, and an improvement although non-significant in hypoxia in ARDS patients. The trial was registered with Clinicaltrials.gov (NCT00314548) and funded by the Pakistan medical research council.
\end{abstract}

Keywords: ARDS; PGE1; Diastolic Dysfunction; Nebulised Prostacyclin; Hypoxia

\section{Introduction}

Adult Respiratory Distress Syndrome (ARDS) is characterized by hypoxemia, low lung compliance, pulmonary hypertension and diffuse radiological infiltrates due to increased microvascular permeability [1]. Clinically it is characterized by severe hypoxia with a $\mathrm{PaO}_{2} / \mathrm{FiO}_{2}$ ratio

\footnotetext{
${ }^{*}$ NIH registered; ClinicalTrials.gov Identifier: NCT00314548. Sponsor: Pakistan Medical Research Council Grant 4-22-3/05/RDC/AKU/3479.

University Ethical Review Committee status: approved. Conflict of interest: none.

An independent not for profit Government funding agency. The authors have no financial interests to disclose.

"Corresponding author.
}

$<200$, Pa pressures $>35 \mathrm{mmHg}$ and possible diastolic dysfunction, reportedly in $25 \%$ patients. The intravenous application of prostacyclin or its stable analogue, PGE1 (alprostadil) has been used to cause a decrease not only of the pulmonary but also of the systemic vascular tone. Aerosolized PGE1 can result in a selective pulmonary vasodilatation without affecting the systemic blood pressure as shown in preliminary studies/case reports [2]. No large prospective trials exist for this type of use of the drug so far. Furthermore, aerosolized PGE1 can improve gas exchange and pulmonary shunt in clinical settings of impaired ventilation/perfusion ratio as occurs in Adult Respiratory Distress Syndrome (ARDS), due to the re- 
distribution of pulmonary blood flow from non-ventilated to ventilated, aerosol accessible lung regions $[3,4]$. Pulmonary hypertension, defined as mean pulmonary artery $(\mathrm{Pa}$ ) pressure of $>25 \mathrm{mmHg}$, is an end point of a variety of conditions. These include primary pulmonary hypertension, post-operative pulmonary hypertension and as a result of increased pulmonary vascular resistance that occurs in ARDS [5-7].

Previous case reports and non randomized studies have indicated that inhalation of aerosolized alprostadil (an analog of prostacyclin) may offer a new life saving strategy in intractable pulmonary hypertension and ARDS [8-10]. Further advantages of inhaled alprostadil are the lack of adverse reactions and toxic side effects; as well as, convenient and more cost effective administration. Other available therapies like Nitric Oxide inhalation are expensive and are also associated with additional side effects, such as thrombocytopenia. Alprostadil is completely metabolized and excretion of metabolites is primarily via the kidneys; the elimination half life is 30 minutes. Inhalation therapy reduces systemic absorption and hence time for elimination [11]. There is an absence of RCTs and systematic reviews on this particular use of the drug for the given specific objectives in literature. The clinical rationale for this study was the untreatable nature of ARDS associated with a high mortality (>30\%) and the incidence of ARDS being $>10 \%$ in our ICU (ICU Quality Indicators Janaury-April 2005-6 [12]). Ours was a trial to show that nebulized PGE1 improves diastolic dysfunction and LVEDP as well as decreases pulmonary hypertension selectively and improves oxygenation in ARDS. Even though the effect of this treatment may be too short lived for a pathophysiological process spanning weeks, we wished to try and find a positive effect of the drug to minimize the associated complications.

\section{Materials and Methods}

This was a single center, prospective, double blind, placebo-controlled, parallel-group study with a balanced randomization conducted in Pakistan. Written approval of Aga Khan University Ethics Review Committee was obtained prior to commencement of the study. Eligible participants were all adults aged 18 or over with ARDS who met the inclusion criteria.

Setting: The study took place in an adult, multi-disciplinary, "open-policy", 11 bedded Intensive Care Unit in a tertiary care hospital of Karachi, Pakistan from May 2006 to February 2008

Patients: A total of 67 patients were enrolled after obtaining informed consent in the patient's native language. Inclusion criteria: After screening all admissions on a daily basis by a Research officer patients were included if they fulfilled the following ARDS criteria: 1) a $\mathrm{PaO}_{2} / \mathrm{FiO}_{2}<$
200 arterial hypoxemia, bilateral infiltrates on chest Xray and a wedge pressure of $<18 \mathrm{mmHg}$ on Swan Ganz parameters if present or if no signs of congestive heart failure were present on echo 2) all patients admitted to the ICU with pulmonary hypertension (Pa systolic pressure of $>35 \mathrm{mmHg}$ ) or 3) all patients in the ICU with post operative pulmonary HTN. Exclusion criteria: Patients were excluded if they had a diagnosis of 1) pulmonary embolus; 2) cor pulmonale; 3) an ejection fraction of $<30 \%$ or a pulmonary artery wedge pressure of $>$ $18 \mathrm{mmHg}$; 4) nonintubated patients; and 5) paediatric patients ( $<18$ years of age). Controls were chosen from selected patients included in the study in a random, blinded manner. Patients were given either a drug or placebo based on the following blinding procedure. After enrolling, the following tests were carried out 1) a trans-thoracic echo to establish pulmonary hypertension and rule out an ejection fraction $<30 \%$ and presence of a pulmonary embolus and 2) routine arterial blood gas and CXR were reviewed to determine ARDS criteria. Transthoracic echo helped determine the left ventricular end diastolic pressures (LVEDP) and diastolic dysfunction. The echocardiographic doppler grades of diastolic function based on mitral inflow patterns are impaired relaxation (grade I) which results from impairments in active relaxation but is not associated with a change in mean left atrial pressure. Further grades of diastolic dysfunction by echocardiographic doppler are grades II [pseudonormal] and III [restrictive] which are associated with an increased mean left atrial pressure [13]. Left ventricular end diastolic pressures (LVEDP) of $<10 \mathrm{mmHg}$ were considered normal, 10 - $15 \mathrm{mmHg}$ were intermediate and $>15 \mathrm{mmHg}$ were considered as increased.

\subsection{Interventions}

Patients were randomly assigned to receive PGE1 or normal saline in a concealed syringe which the bedside nurse dispensed as a nebulizer in $5 \mathrm{ml}$ of saline over 30 minutes. The drug alpostadil was nebulized continuously at 20 micrograms in normal saline over 30 minutes [8,13]. Drug administration was carried out within one hour of the first transthoracic echo and arterial blood gases. Patients in the control and intervention groups received usual care at the discretion of their intensivist. All cost of investigations and drug were borne by the grant obtained for this study prior to commencement. Baseline $\mathrm{PaO}_{2} /$ $\mathrm{FiO}_{2}$ ratio was obtained from the arterial blood gas analysis (ABG) documented in the flowsheet which is routinely carried out in the ICU on all ventilated patients. Baseline diastolic function, left ventricular end diastolic pressure and $\mathrm{Pa}$ pressure was obtained on echocardiogram in all patients. After the enrolment with a $\mathrm{PaO}_{2} /$ $\mathrm{FiO}_{2}$ ratio less than 200 and a Pa pressure greater than 35 
mm Hg, PGE1 or placebo was nebulised in 5 cc of normal saline for thirty minutes by the bedside nurse using 5 $\mathrm{L}$ of oxygen. Jet nebulizers were used connected by tubing to a compressor, that usually causes compressed air or oxygen to flow at high velocity through a liquid medicine to turn it into an aerosol, which is then inhaled by the patient. During this time the haemodynamics and saturation was closely monitored and the patient was excluded if there was instability or if hypoxia worsened. A repeat $\mathrm{ABG}$ and echocardiogram was performed at the bedside after one hour of drug administration to record the "after drug" parameters.

\subsection{Standardization of Care}

Standardization of treatment modalities have been incorporated in our methodology with regard to ventilatory support, inotropic support and fluid management. A general range of parameters for ARDS and end goal directed therapy of the Surviving Sepsis campaign were maintained, keeping in mind the recommendations of the Society of Critical Care medicine and the Acute Respiratory Distress Syndrome Network [14-16]. Once enrolled, the patients were managed by the ICU Consultant in consultation with the primary physician along the following lines: any ventilatory mode could be used as long as peak airway pressures (PAw) were maintained under 35 $\mathrm{mmHg}$. Peep range was between $8-14 \mathrm{mmH}$ and tidal volumes of $6 \mathrm{cc} / \mathrm{kg}$ were used. Inotropic support, if needed, was maintained by using intravenous infusion of Dopamine (5 - $20 \mathrm{mics} / \mathrm{kg} / \mathrm{min}$, a positive inotrope (dopaminergic, alpha and beta agonist activity) and norepinephrine (0.1 - $0.5 \mathrm{mics} / \mathrm{kg} / \mathrm{min}$ ) a vasopressor (alpha agonist) to maintain a mean arterial pressure of $>60 \mathrm{mmHg}$. CVP pressures of $8-12 \mathrm{~cm} \mathrm{H}_{2} \mathrm{O}$ and an adequate urine output of $>0.5 \mathrm{cc} / \mathrm{kg} / \mathrm{hr}$ were the goal of fluid management.

\subsection{Outcome Measures}

The primary end point with respect to efficacy of inhaled PGE1 in ARDS was the proportion of patients achieving a 25\% improvement in diastolic dysfunction, LVEDP, Pa pressures and $\mathrm{PaO}_{2} / \mathrm{FiO}_{2}$ ratio from baseline as measured by a repeat transthoracic echo and arterial blood gas analysis thirty minutes after the drug was administered.

\subsection{Randomisation and Allocation Concealment}

Independent pharmacists dispensed either active or placebo drug according to a computer generated randomization list. The PGE1 and placebo were dispensed from pharmacy in a syringe form concealed with an aluminium foil. They were consecutively numbered for each patient according to the randomization schedule.

\subsection{Blinding and Data Collection}

Each patient after enrolment was assigned a serial number consecutively after meeting the inclusion criteria reviewed by the principal investigator and research assistant. After the necessary workup and consent, drug was obtained from the AKUH Pharmacy. All investigators, staff and patients were kept masked to outcome measurements and allocation. Data was collected by the research assistant on a data collection form. At the end of trial the serial numbers of the patients were matched to the delivery chart of the pharmacy to see whether they obtained PGE1 or placebo. Variables recorded are given in Table 1.

\subsection{Statistical Methods}

Epi-info version 1.1 was used to calculate sample size. Assuming a total population of over 15 million (2010 Demographic Survey of Pakistan) of all age groups, and taking the incidence of 20\% ( \pm 105 bound of error) of Pulmonary hypertension and ARDS at $95 \%$ confidence interval, we extricated a sample size to be 60 cases, to achieve a power of $80 \%$ and a significance of $5 \%(\alpha=$ $0.05)$ [17]. We predicted a $10 \%$ attrition rate. The primary end point was change in diastolic dysfunction, LVEDP, Pa pressures and $\mathrm{PaO}_{2} / \mathrm{FiO}_{2}$ ratio during the study in the intention-to-treat principle. Descriptive statistics were performed. An independent samples Student's t-test was used to test for significant differences at baseline before drug and between groups after drug. Group means of cases and controls were then calculated and compared using a repeated measures analysis of variance (ANOVA) procedure.

\section{Results}

Two hundered consecutive patients were screened between May 2006 and Feb 2008. Twenty patients refused to enroll. Seventy two patients were enrolled after meeting the inclusion criteria and after informed consent was obtained from the next of kin as well as permission obtained from the primary physician. Five patients were excluded later as they expired before drug administration. Finally, 34 were randomized to the PGE1 group and 33 to the placebo group. The characteristics of the final 67 patients are summarized in Table 1, including the age, gender, baseline diastolic dysfunction, LVEDP, Pa pressure and $\mathrm{PaO}_{2} / \mathrm{FiO}_{2}$ ratio. At baseline, mean diastolic dysfunction was grade II, with a mean LVEDP of $>15$ and the $\mathrm{PaO}_{2} / \mathrm{FiO}_{2}$ ratio was $148.38 \pm 60.05$ with a mean pulmonary artery pressure of $81.35 \pm 16.91$. Patients were well matched for age, type of admission, illness severity, and other clinical characteristics. Figure 1 illustrates 
Table 1. Comparison between the two groups pre-intervention at baseline (Data are means \% and 95\% CI).

\begin{tabular}{|c|c|c|c|}
\hline & PGE1 group $(n=34)$ & Placebo group $(\mathrm{n}=33$ ) & $\mathrm{p}$ value \\
\hline Age (years) & $48.22(41.63$ - 54.81) & 48.21 (42.55 - 53.87) & 0.056 \\
\hline APACHE II score & $24(22.82-25.18)$ & $21(19.23-22.70)$ & \\
\hline Males (\%) & $62.5(45.12$ - 77.09) & $71.9(54.47$ - 84.40) & 0.42 \\
\hline Females (\%) & 37.5 (22.90 - 54.87) & $28.1(15.59$ - 45.52) & \\
\hline \multicolumn{4}{|l|}{ Diastolic dysfunction } \\
\hline $\mathrm{Nl}$ & 0 & $3.0(0.72-15.32)$ & 0.10 \\
\hline I & $14.7(6.56-30.25)$ & $15.2(6.76-31.05)$ & \\
\hline IA & 58.8 (42.11 - 73.67) & $48.5(32.42$ - 64.87) & \\
\hline II & $2.9(0.69-14.91)$ & $21.2(10.74-37.89)$ & \\
\hline \multicolumn{4}{|l|}{ LVEDP (mmHg) } \\
\hline$<10$ & $3.8(0.91$ - 18.97) & $3.4(0.08-17.21)$ & 0.97 \\
\hline $10-15$ & $19.2(8.62$ - 38.08) & $17.2(7.71-34.72)$ & \\
\hline$>15$ & 76.9 (57.74 - 88.88) & $79.3(61.43$ - 90.06) & \\
\hline $\mathrm{PaO}_{2} / \mathrm{FiO}_{2}$ baseline & 141.15 (120.77 - 161.53) & $163.41(140.84-185.98)$ & 0.17 \\
\hline Pa pressure baseline (mmHg) & 81.99 (76.5 - 87.48) & 80.40 (71.25 - 89.55) & 0.77 \\
\hline
\end{tabular}

the trial profile.

The primary analysis was intention-to-treat and involved all patients who were randomly. Group means of cases and controls were calculated and compared using a repeated measures analysis of variance (ANOVA) model which that, when separately considered, there was a significant difference in Pa pressures after the drug administration between the cases and controls $(p=0.04)$. There was a marginal non significant difference in the $\mathrm{PaO}_{2}$ / $\mathrm{FiO}_{2}$ ratio after the drug between cases and controls ( $\mathrm{p}=$ 0.21). Table 2 denotes the comparison of the means pre and post intervention by case control groups. Inhaled PGE1 was followed by an improvement in diastolic dysfunction (grade $\mathrm{I}, \mathrm{p}=0.001$ ) with a resulting improvement in LVEDP (12 $\pm 2, \mathrm{p}=0.001)$ as well as Pa pressures $(97.09 \pm 30.06, \mathrm{p}=0.04)$ and a non significant improvement in $\mathrm{PaO}_{2} / \mathrm{FiO}_{2}$ ratio (161.45 \pm 77.52, $\mathrm{p}=0.21$ ) as shown in Figures 2 and $\mathbf{3}$ and Table 3 . There were no patients who had to be removed from the trial for hemodynamic instability or worsening hypoxia. There were also no other complications observed.

\section{Discussion}

In our study we found that PGE1 when nebulised in such critically ill patients is safe and effective in decreasing the diastolic dysfunction, left ventricular end diastolic pressures, and pulmonary artery pressures which contributes significantly to the morbidity and mortality of

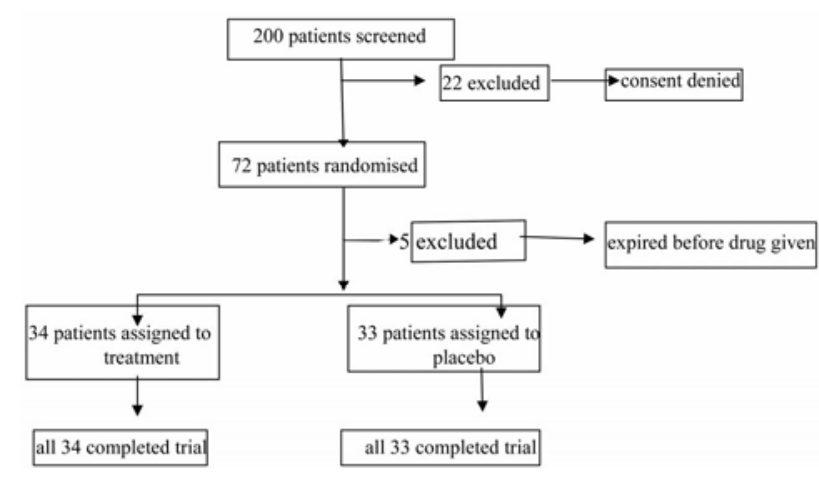

Figure 1. Trial profile.

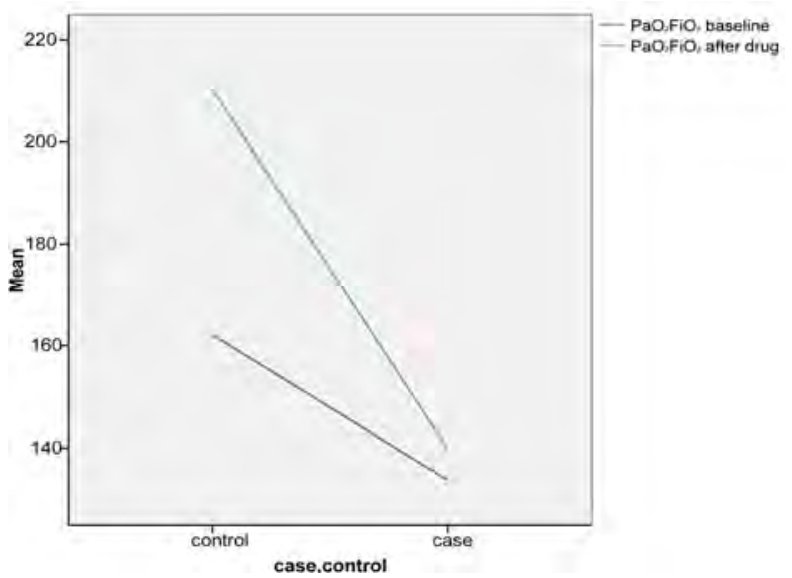

Figure 2. Comparison of $\mathrm{P} / \mathrm{F}$ ratio in cases and controls before and after drug. 
Table 2. Comparison between the two groups post intervention (Data are means \% and 95\% CI).

\begin{tabular}{cccc}
\hline Endpoint & PGE $1(\mathrm{n}=34)$ & Placebo $(\mathrm{n}=33)$ & p value \\
\hline Mean diastolic dysfunction & Grade I & Grade I & 0.001 \\
LVEDP & & & 0.001 \\
$<10$ & $19.2(8.62-38.08)$ & $27.6(14.73-45.88)$ & \\
$10-15$ & $57.5(38.79-74.52)$ & $48.3(31.29-65.67)$ & 0.04 \\
$>15$ & $23.1(11.11-42.25)$ & $24.1(12.27-42.28)$ & 0.21 \\
Pa pressure & $82.66(75.12-90.2)$ & $97.09(86.67-107.51)$ & $186.80(162.87-210.73)$ \\
$\mathrm{PaO}_{2} / \mathrm{FiO}_{2}$ & $161.45(134.59-188.31)$ & &
\end{tabular}

Table 3. Comparison between pre- and post-nebulisation parameters (Data are means \% and 95\% CI).

\begin{tabular}{cccc}
\hline Characteristics & PGE1 group ( $\mathrm{n}=34)$ & Placebo group ( $\mathrm{n}=33)$ & p value \\
\hline $\mathrm{PaO}_{2} \mathrm{FiO}_{2}$ baseline, Mean (SD) & $148.38 \pm 60.05$ & $162.84 \pm 66.20$ & 0.52 \\
$\mathrm{PaO}_{2} \mathrm{FiO}_{2}$ after drug, Mean (SD) & $161.45 \pm 77.52$ & $186.80 \pm 69.07$ & 0.12 \\
Pa pressure baseline, Mean (SD) & $81.35 \pm 16.91$ & $80.38 \pm 26.86$ & $97.09 \pm 30.06$ \\
Pa pressure after drug, Mean (SD) & $82.66 \pm 21.77$ & $17.83 \pm 3.79$ & 0.36 \\
LVEDP baseline & $18.27 \pm 3.45$ & $12.90 \pm 3.24$ & \\
LVEDP after drug & $14.12 \pm 3.73$ & & \\
\hline
\end{tabular}

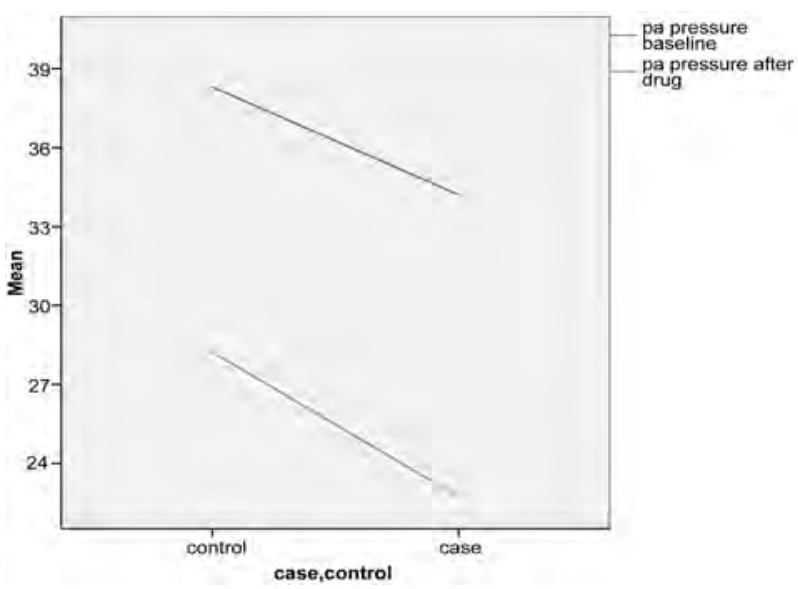

Figure 3. Comparison of PA pressures in cases and controls at baseline and after drug.

such patients. We, however, could not demonstrate significance in the change in $\mathrm{PaO}_{2} / \mathrm{FiO}_{2}$ ratio or hypoxia, even though there was a rising trend in this post drug administration. These findings are of clinical relevance because ARDS is a common cause of hypoxia, poor lung compliance and pulmonary hypertension in the ICU, all leading to multi organ failure, and in conjunction with sepsis (which usually is a precursor of ARDS) $[18,19]$ is associated with high morbidity and mortality rates [20]. The acute respiratory distress syndrome (ARDS) is characterized by a ventilation-perfusion mismatch resulting from the non-ventilated atelectatic lung areas which results in a intrapulmonary right-to-left shunt with the consequence of severe arterial hypoxemia. The application of inhaled PGE1 is a therapeutic option to selectively influence pulmonary blood flow in order to improve arterial oxygenation and to decrease pulmonary artery pressure without relevant systemic side effects. Although there are case reports present in literature, no randomized controlled trials have been carried out to our knowledge to demonstrate a benefit in patient populations covering the entire severity range of acute lung injury and diastolic dysfunction parameters. Small studies have shown an improvement in RV function in ARDS with inhaled nitric oxide (iNO) $[21,22]$. We therefore suggest that based on our results PGE1 represents a feasible rescue treatment for ARDS patients with diastolic dysfunction, raised LVEDP and increased pulmonary artery pressures and is, therefore, an important option for diastolic heart failure occurring in the presence of ARDS until further larger trials are carried out and other alternative treatments are observed. Studies looking at inhaled nitric oxide (iNO) suggest a large cost involved as well as use only in specialized centers and therefore may not be a realistic option for our population $[23,24]$.

This is the first time such a study has been conducted and it showed robustly the efficacy of the drug as well as generalisability of effect, albeit for a short term.

The small cohort due to decreased rates of ARDS after 
commencement of the study in our ICU could be taken as a potential limitation. The study could enroll fewer than expected patients due to the drop in the incidence of ARDS in our ICU during the course of the study. One can speculate as to the causes of this, one being better ventilatory management of the disease or better control of severe sepsis due to the Surviving sepsis campaign [18].

Also, we were not observing the survival or length of stay in the ICU of these patients which is also a potential limitation of the study. Based on the available observations, we found that inhaled PGE1 in patients with ARDS safe and effective in reducing pulmonary artery pressures. However, results of improvement in hypoxia, although promising was non significant. We would advocate larger multi center trials to look at the effect on hypoxia as well as patient centered end points such as survival and length of stay outcomes, as well as ventilatory requirements.

\section{Acknowledgements}

We wish to especially thank Drs. Saad Khan, Adeel Reza, Rafay for data collection and entry. Dr. Saulat Fatimi for his intellectual input; Ms Safia Awan for data analysis; Ms Roshan Manasia, Zohra Pirani RNs for clinical facilitation, Drs. Sajid Dhakum and Qamar ul Hoda for allowing this study to proceed in the echo department and the ICU. We wish to thank all ICU, as well as pharmacy and cardiology staff for the conduct of this study.

\section{REFERENCES}

[1] G. Domenighetti, H. Stricker, et al., "Nebulized Prostacyclin in ARDS: Impact of Primary and Secondary Disease on Gas Exchange Response," Critical Care Medicine, Vol. 29, No. 1, 2001, pp. 57-62. doi:10.1097/00003246-200101000-00015

[2] S. Macherndl, M. Kneussl, et al., "Long Term Treatment of Pulmonary Hypertension with Aerosolized Iloprost," European Respiratory Journal, Vol. 17, No. 1, 2001, pp. 8-13. doi:10.1183/09031936.01.17100080

[3] M. Max and R. Rossaint, "Inhaled Prostacyclin in the Treatment of Pulmonary Hypertension,” European Respiratory Journal, Vol. 158, No. S1, 1999, pp. S23-S26. doi:10.1007/PL00014316

[4] M. Hoeper, H. Olschewski, et al., "A Comparison of the Acute Hemodynamic Effects of Inhaled Nitric Oxide and Aerosolized Iloprost in Primary Pulmonary Hypertension. German PPH Study Group,” Journal of the American College of Cardiology, Vol. 35, No. 1, 2000, pp. 176-182. doi:10.1016/S0735-1097(99)00494-5

[5] H. Olschewski, H. Ghofrani, et al., "Inhaled Iloprost to Treat Severe Pulmonary Hypertension. An Uncontrolled Trial. German PPH Study Group," Annals of International Medicine, Vol. 132, No. 6, 2000, pp. 435-443.
[6] N. Galie and A. Manes, "Medical Therapy of Pulmonary Hypertension. The Prostacyclins," Clinics in Chest Medicine, Vol. 22, No. 3, 2001, pp. 529-537. doi:10.1016/S0272-5231(05)70289-6

[7] G. Raimondi, "Gas Exchange in Acute Respiratory Distress Syndrome,” Medicina (Buenos Aires), 2003, pp. 157164.

[8] K. Theodoraki, P. Rellia, et al., "Inhaled Iloprost Controls Pul Hypertension after Cardiopul Bypass,” Canadian Journal of Anesthesia, Vol. 49, No. 9, 2002, pp. 963-967. doi:10.1007/BF03016884

[9] T. J. Lasserson, et al., "Prostacyclin for Pulmonry Hypertension," Cochrane Database Systematic Review, Vol. 2, 2003, Article ID: CD002994.

[10] R. T. Schermuly, A. Schulz, et al., "Pharmacokinetics and Metabolism of Infused Versus Inhaled Iloprost in Isolated Rabbit Lungs," Journal of Pharmacology and Experimental Therapeutics, Vol. 303, No. 2, 2002, pp. 741-745. doi:10.1124/jpet.303.2.741

[11] T. Gessler, T. Schmehl, et al., "Aerosolized Vasodilators in Pulmonary Hypertension,” Journal of Aerosological Medicine, Vol. 15, No. 2, 2002, pp. 117-122.

[12] “ICU Quality Indicators 2002-2003,” Aga Khan University, Karachi.

[13] M. R. Zile and D. L. Brutsaert, "New Concepts in Diastolic Dysfunction and Diastolic Heart Failure. Part II: Causal Mechanisms and Treatment," Circulation, Vol. 105, 2002, pp. 1503-1508. doi:10.1161/hc1202.105290

[14] A. Sablotzki, T. Hentschel, et al., "Hemodynamic Effects of Inhaled Effects of Inhaled Aerosolized Iloprost and Inhaled Nitric Oxide in Heart Transplant Candidates with Elevated Pulmonary Vascular Resistance,” European Journal of Cardiothoracic Surgery, Vol. 22, No. 5, 2002, pp. 746-752. doi:10.1016/S1010-7940(02)00488-8

[15] Lusardi, et al., "2001 Open Forum at International Resp Congress: The Annual Convention and Exhibition for Respiratory Care, 2001, San Antonio,” Respiratory Care, Vol. 46, No. 10, 2001, pp. 1065-1070.

[16] R. Brower, "Mechanical Ventilation in Acute Lung Injury and ARDS. Tidal volume Reduction," Critical Care Clinics, Vol. 18, No. 1, 2002, pp. 1-13.

[17] R. G. Brower, L. B. Ware, Y. Berthiaume and M. A. Matthay, "Treatment of ARDS," Chest, Vol. 120, No. 4, 2001, pp. 1347-1367. doi:10.1378/chest.120.4.1347

[18] E. James, II. Bartlett, J. W. Kotrlik and C. C. Higgins, "Organizational Research: Determining Appropriate Sample Size in Survey Research,” Information Technology, Learning and Performance Journal, Vol. 19, No. 1, 2001.

[19] T. Busch, S. Bercker, et al., "Inhaled Nitric Oxide for Rescue Treatment of Refractory Hypoxemia in ARDS Patients," Anasthesiol Intensivmed Notfallmed Schmerzther, Vol. 43, No. 11-12, 2008, pp. 778-783. doi:10.1055/s-0028-1104618

[20] Z. G. Wei, B. Zhong, J. Ji and X. Yi, “Guidelines for Management of Acute Lung Injury/Acute Respiratory Distress Syndrome," The Chinese Society of Critical Care Medicine, Vol. 218, No. 12, 2006, pp. 706-710. 
[21] J. M. Camamo, R. H. McCoy, et al., "Retrospective Evaluation of Inhaled Prostaglandins in Patients with Acute Respiratory Distress Syndrome,” Pharmacotherapy, Vol. 25, No. 2, 2005, pp. 184-190. doi:10.1592/phco.25.2.184.56952

[22] J. Cranshaw, M. J. Griffiths, et al., "The Pulmonary Physician in Critical Care. Part 9: Non-Ventilatory Strategies in ARDS,” Thorax, Vol. 57, No. 9, 2002, pp. 823-829. doi:10.1136/thorax.57.9.823
[23] K. F. Schulz, D. G. Altman and D. Moher, "CONSORT 2010. Statement: Updated Guidelines for Reporting Parallel Group Randomised Trials,” Annals of International Medicine, Vol. 152, 2010.

[24] L. Fierobe, F. Brunet, J. F. Dhainut, et al., "Effect of Inhaled Nitric Oxide on Right Ventricular Function in Adult Respiratory Distress Syndrome," American Journal of Respiratory and Critical Care Medicine, Vol. 151, No. 5, 1995, pp. 1414-1419.

\section{Abbreviations}

PA: Pulmonary Artery

LVEDP: Left Ventricular End Diastolic Pressure

ARDS: Adult Respiratory Distress Syndrome

PGE1: Prostaglandin E1

$\mathrm{PaO}_{2}$ : Partial Pressure of Oxygen

$\mathrm{FiO}_{2}$ : Fraction of Inhaled Oxygen

iNO: inhaled Nitric Oxide 